\title{
Optimum Path Connectivity and Coverage in WSN to Increase Network Lifetime: A Review
}

\author{
Kishu Pathania \\ M.Tech, \\ ITM University \\ Gurgaon, Haryana \\ India
}

\author{
Shilpa Mahajan \\ Assistance Professor, \\ ITM University \\ Gurgaon, Haryana \\ India
}

\begin{abstract}
As we all know, this age has become the age full of many different technologies which are helping each and every personality in their daily routine directly or indirectly. One of these technologies wireless sensor network has emerged in many areas and has gained a large popularity because of its great capabilities and functionalities. Wireless sensor network shortly named as WSN, is a network made by grouping some or many sensor nodes, technically known as motes, which are able to make communication with each other within the network range for performing many different tasks when needed and colleting the necessary information for achieving the purposed goal of that network and then forward that complete information to the base station of that network, technically called sink. In recent years WSN has made its presence in most of the areas for example military applications, medical applications and industrial areas. As WSN is being widely used but there is still many problems are present in WSN. Some problems of WSN are maximum target coverage problem, most energy efficient network and fault tolerant network. For effective communication it is necessary to cover most of the targets and also use optimum path to connect nodes so that network lifetime could be increased. Main concern of target coverage problem is the random deployment of the sensor network for supervising the specific targets for rising the life span of the network it also dictates that how much efficiently the monitoring and sensor tracking of any sensor network works.. To increase the lifetime of the network an optimal node deployment scheme is required that would minimize the cost of the network, reduce the energy consumption and resilient to node. In this paper we have proposed a system that can be used to solve all these issues after reviewing some previous researches in this field. In this work we will use a hybrid approach of merging some important algorithms and approaches to design a system that can solve these issues. Our system will work in two phases: First, Genetic Algorithm (GA) is applied to achieve optimum result and second, Support Vector Machine (SVM) is used to achieve a fault tolerant energy efficient network. SVM is used to detect intrusions like black hole attack and selective forwarding attack. In our approach both the phases will work in parallel for generating most efficient optimum path.
\end{abstract}

\section{General Terms}

Wireless Sensor Network, Genetic Algorithm, Coverage Issues.

\section{Keywords}

WSN, Optimum path, GA, SVM (Support Vector Machine).

\section{INTRODUCTION}

We can give definition of a wireless sensor network as a collection of two or more mobile or stationary sensor nodes which forms a temporary network without any particular infrastructure or central administration. These sensor nodes are mainly called motes. A wireless sensor network $[1,2,3]$ may consist of many sensor nodes from hundreds to thousands which are communicating with each other via wireless channel for sharing their information for different purposes. Wireless sensor nodes have the ability to gather the information from the area where they are located and after performing the particular calculation of that data transmit that information to a base station via wireless medium. The sensor nodes in the wireless sensor network are mainly small battery powered tint sensor devices which are able to perform sensing tasks like environmental conditions, temperature, movement in the area and atmospheric pressure and many more. A node is usually prepared with several components like a sensor unit, ADC (analog to digital converter), CPU(central processing unit), a power source (commonly a small battery) and a transceiver (transmitter and receiver) device unit [1].. Each wireless sensor network may consist one or more base stations called sink for collecting and storing the data sensed by the sensor nodes in the network. A typical WSN is shown in figure 1.

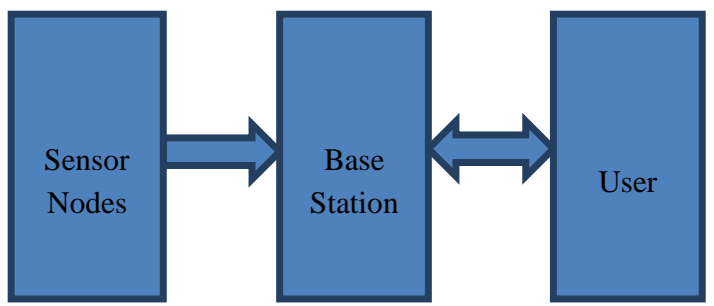

Figure 1: Typical WSN Architecture

Day by day rapid advancement and development of new technologies in wireless sensor network and new researches in micro-sensor device has accelerated the use of WSN in many application areas such as industrial monitoring, military applications, medical applications, forest fire detection and many more. As the technology is advancing in all areas of wireless sensor network, there is a need of more suitable and more efficient routing scheme which is able to enhance the life time of the wireless sensor network by reducing the power consumption and choosing the most suitable shortest path for the delivery of data packets. Limited amount of power source, dynamically changing network topology, mobile or stationary both types of nodes, communication broadcasting in short range, deployment at larger scale and multi-hop routing, these are some basic and common characteristics of a wireless sensor network [1]. 
Efficient and accurate resource management with reliable QoS is an essential requirement in a wireless sensor network. However, due to less resource constraints and hostile environmental conditions, it is difficult to design efficient node deployment scheme which has the ability to minify the overall network expenses, consumable energy reduction with the reduction in computing steps and reduce the probability of attacks on the network is very difficult because of the lower number of available resources and inhospitable circumstances

Coverage problem $[3,4,5,6,7]$ is very crucial operative issue of WSN which describes that a sensing network is how much capable of covering sensing nodes and efficiency of covered sets of sensing nodes. Coverage issue mainly interacts to quality of service parameters of networks to insure that an active sensing network must be attended and checked by minimum sole sensing node. Because of the limitation in battery, how to enhance the network life span is a significant concern in wireless sensor networks. For coverage issue, lifetime is the time period that all the targets or the region is constantly enclosed. Target Coverage issue is troubled with the random deployment of sensor nodes for supervising the specific targets for maximum period. In Target Coverage, each Target is monitored by at least one sensor node. Coverage issue is very crucial operative issue in wireless sensor network which disposes that a sensing network is how much capable of covering sensing nodes and efficiency of covered sets of sensing nodes [7]. The coverage issue directly interacts to quality of service parameter to ensure that an active sensing network must be attended and checked by minimum sole sensing node.

In addition to coverage issue providing better connectivity between sensing nodes and also keep this connectivity maintained during the packet transfer is also the active issue in the field of wireless sensor network. Direct and indirect communication between any active nodes for the efficient and reliable transmission of data packets is the working feature of connectivity [3]. When there is no connectivity between nodes of the network then there is no possibility of any type of communication process between nodes and the base station, this leads to no data transmission and reception to base station and to other active nodes. Because of all this the combined study of the coverage and connectivity issues of wireless sensor network has becomes the active crucial issue [8].

Sensing network design for finding a most optimal connection between randomly deployed nodes we can use DFS based routing schemes with genetic algorithms for reducing the repetitive nodes, route requests, overhead maintenance cost, consumable unit and also increase the sensing range of the network. Optimized target coverage is an significant issue in the wireless sensor networks [8].

The most extensively used structure of evolutionary computation; the Genetic Algorithms (GAs) have confirmed to be a most successful meta heuristic method for many NP-complete optimization problems [6]. Genetic algorithm (GA) is a search method to find approximate solutions to combinatorial optimization problems. It is difficult to obtain a solution for finding a better coverage for a k-covered and connected sensor network deployment. The space of all feasible solutions space can be quite huge.
Another issue in WSN is security of nodes or failure detection in the network. In most cases, the sensor nodes are randomly distributed in no security manner, and it is easy for nodes to be captured and manipulated by attackers. In recent years, WSN security problem has become the important research area to scholars. The author in [9] summarized the types of attacks in WSN, such as black hole attack, selective forwarding attack and so on

Support Vector Machine (SVM) is most widely used classification methods in WSN. It has peculiar advantages in small sample, high dimension pattern recognition and nonlinear problems $[10,11]$. In this paper we focus on adapting a simple SVM classification based intrusion detection system to notice a precise spectrum of malicious DoS attacks such as selective forwarding attack that might be introduced against a Wireless sensor networks. This IDS uses routing information local to the base station of the network and raises alarms based on the 2D feature vector (1) hop count. (2) bandwidth.Data patterns are classified using a one-class SVM classifier

The remainder of this article is structured as shown; in section 2 discusses about some literature works done on the topics of GA and SVM. In section 3 we discuss important algorithms, in section 4 we introduced proposed work based on the literature review. Finally, in section 5 we conclude our research work.

\section{LITURATURE REVIEW}

In this section we will provide the reviews of some previous researches which are related to our work and necessary to be discussed here for better understanding of our work.

In [2], authors have described the energy efficiency issue, connectivity and coverage problem of WSN and provide a solution for these problems which is based on genetic algorithms. They have described that in large scale reconnaissance system the life span of wireless sensor network system is the time duration that all targets can be enclosed and target coverage problem is one of the issues to increase the life period of wireless sensor network. They have proposed that these problems can be resolved by scheduling the sensors activity by maintaining least number of nodes active at moment where sensors from the active sets are supervising all targets and responsible to broadcast the data to the base station and the rest of the nodes are in sleep mode. In this paper they have proposed a GA which uses least number of active nodes by forming cover sets by dividing all the deployed sensors in to mutually elite subsets of sensors or set cover such that each set of can cover all targets. After discovering the set cover they may work by turns means at one time just one set will active and remaining will be in sleep mode by designing heuristics that effectively calculate the set covers hence life duration of WSNs can be interrupted by by discovering more sensor set

In [3], Authors presents a detailed survey of WSN based on coverage and connectivity problem. They proposed some terms and concepts as the solution of these problems and then shoe how they can be used in various research works.

In [4], researchers have proposed a new scheme called Ntarget coverage for target coverage problem in WSN. They have defined a hybrid approach of Depth First Search (DFS) along with Genetic Algorithm (GA) to extend lifetime of the network. They have described a two layer working architecture in which the first layer was performed by DFS so 
that the minimum connectivity coverage could be identified respective to each target and in second layer the genetics was implemented so that the optimized solution could be derived.

In [5], researchers of the work have described an energy efficient target coverage scheme for WSN which was based on modified Ant Colony Algorithm. They have discussed the target coverage problem in detail and also have provided a solution for that. They have also stated that the problem can be resolved by choosing least number of functioning nodes that would cover all the targets (same statement is also given in [2]). They have proposed a new approach of selecting minimum number of working nodes by modified ant colony approach.

Also in [8],authors have discussed target coverage and connectivity problem of WSN. They have implemented DFS along with GA to find out optimal node deployment strategy for minimizing the network configuration cost energy consumption and communication overhead. In this paper researchers had projected a genetic based depth-first search algorithm for achieving optimum connectivity. They have also shown some simulation results based on their proposed scheme.

In [9], authors have discussed some most important attacks of WSN. They have provided a complete analysis of some major attack of WSN like DOS attack, black hole attack, and selective forwarding attack and so on.

In [10], researchers have proposed a multi-class method based on Error Correcting Output Codes algorithm in order to get better performance of attack recognition in Wireless Sensor Networks. In this paper aiming to enhance the accuracy of attack detection, the multi-class method was constructed with Hadamard matrix and two-class Support Vector Machines (SVM) and to minimize the complexity of the algorithm, sparse coding method was implemented in this paper. They have used SVM for fault detection in WSN.

In [11], the use of SVM's kernel function made the nonlinear flow data collected by sensor nodes to be mapped to a highdimensional feature space, which greatly reduces the algorithm complexity, and effectively overcomes the "dimension disaster" problems which often appears in artificial neural networks.

In [13] authors have proposed a scheme based on SVM for intrusion detection which was suitable for low bandwidth and small packets situations in wireless sensor networks.

Thus, it is clear that energy efficiency, target coverage and connectivity topics have always been the research interest for many researchers. That's why we have decided to find out a common solution that can provide an energy efficient fault tolerant wireless sensor network with optimum connectivity.

\section{IMPORTANT ALGORITHMS}

Genetic Algorithm (GA): The genetic algorithm is the searching strategy to find optimal solution of a given problem and it also provides an approach to find out the solution of the constrained and unconstrained type of optimization problems which are mainly based on the node selection. All genetic algorithms applies some modifications on a predefined population value of individual problem solutions. The GA randomly selects different individuals from the currently defined population to be parents and applies these credentials to generate the children for the next population sets. After the successful generation of children, the population "evolves" towards an optimum solution. The genetic algorithm has the capability to provide the solution for different types of optimization problems which are suitable for standard optimization algorithms. Mainly three basic types of rules are defined in the genetic algorithm for generating the next population set by using the current initial population. The very first rule is the selection rule which selects the parents and it help to generate afterwards population set. Second is crossover rules which form children for next population set by combining two parents. Third rule is mutation rule those are applied for unsystematic changes to individual parents to form children.

\section{Terminologies of $G A$}

Fitness Function: The fitness function is the function need to be optimized. The objective function is standard optimization algorithm. The toolbox tries to find the smallest of the fitness function.

Individuals: An individual is any point to which you can apply the fitness function. The value generated by the fitness function is its score. An individual is sometimes referred to as a genome and the vector entries of an individual referred as genes. It is also defined as representation. Chromosome can be bit string, real numbers, lists of rules etc. Here the chromosomes are represent in the bit string either 0 or 1 , if chromosome is 1 it means the sensor node is present in wake up state and if chromosome value is 0 it means the sensor node is in sleep state.

\begin{tabular}{|l|l|l|l|l|l|l|l|l|l|l|l|l|l|l|l|}
\hline 0 & 1 & 0 & 1 & 0 & 1 & 1 & 0 & 0 & 1 & 1 & 0 & 1 & 1 & 1 & 0 \\
\hline
\end{tabular}

Figure 3: Chromosome Representation

Populations and Generations: We can say population is an array of individuals.For example, suppose the population size is 1000 and 4 variables are there in the fitness function and represent the population by a 1000 by 4 matrixes. The similar individual can appear more than one time in the population.

Fitness Values: The fitness value of an individual is the value of the fitness function for that individual. The best fitness value for a population is the least fitness value for any individual in the population because the toolbox finds the minimum of the fitness function.

Support Vector Machine: SVMs (Support Vector Machine) are mainly known as the class of machine learning algorithms [13]. These machines are mainly the learning models which are used for analyzing and recognizing the patterns and these SVMs are mainly designed for the purpose of binary linear classification and regression analysis. Now a days the SVMs has expanded their use in many areas in the form of regression analysis, density estimation, one-class and multi-class. In most previous years after the origination of SVMs these has been used for different tasks like handwritten isolated digit recognition, different object recognition, particular face detection from an image and many more. Thus SVMs becomes most familiar tools to be used for dealing with most complex type and non-linear form of problems in a very well formed and structured way.

This research mainly focuses only on the one-class type of support vector machines which are used for analyzing and detecting the selective forwarding type of intrusions performed over a wireless sensor network. The selection of this type of support vector machine has been done because the form of any type of intrusion is not pre-known to us, so it is 
easy to construct any type of intrusion and it becomes easy to get the accurate and effective representation of the intrusion performed over the sensing network.

The SVM can be described in the following manner: In the priory step the SVM builds up the hyper plane or a bunch of many hyper planes in a very high infinite dimensional space and these hyper planes are further used for different tasks like regression, pattern recognition, classification and many more. After the hyper plane generation the separation is performed and an effective separation can be achieved when the chosen hyper plane has the longest distance to their nearest training data set point of any type of class and this is known as the functional margin of classification. But in most general terms it is said that when there is lower margin rate then the generalization error of classification also becomes lower

After all this it is stated that the main authentic classification problem might be designed in a finite dimensional space and primarily it occurs that the discriminating sets are not linearly separable in the finite dimensional space. Because of this type of problem, a solution was proposed which dictates to map the initial finite dimensional space into a much higher infinite dimensional space assuming that it can make the separation easy in the final achieved space. For minimizing the load of the calculation and computation to a reasonable point, the mapping schemes used by the support vector machine's algorithms, are structured to make sure that the dot products can be simply calculated in the terms of the variables in that initial space, and it can be done by initializing these dot products in the form of a kernel function $\mathrm{K}(\mathrm{x}, \mathrm{y})$ to match the problem statement. After that the hyper planes designed in the higher dimensional space are initialized in the form of the set of points which returns a constant value after the inner product of that with any vector value that space. The vectors describing the hyper planes can be selected to be linear combinations with parameters $\alpha_{i}$ of images of feature vectors that arise in the data base. With this choice of a hyper plane, the points $\mathrm{x}$ in the feature space that are mapped into the hyper planes are defined by the given relation: $\sum_{\mathrm{i}} \alpha_{\mathrm{i}} \mathrm{K}\left(\mathrm{x}_{\mathrm{i}}, \mathrm{x}\right)=$ constants. It is also to be notified that when the value of variable $\mathrm{y}$ increases from point $\mathrm{x}_{\mathrm{i}}$, the values of kernel function $\mathrm{K}(\mathrm{x}, \mathrm{y})$ decreases and every variable used in the computation of the vector sum determines the level of nearness of the test point $\mathrm{x}$ to the corresponding data base point $\mathrm{x}_{\mathrm{i}}$.

\section{PROPOSED WORK}

After analyzing some important literature works done in WSN for coverage and connectivity issues, we have find out some important points than could be helpful in finding out a common solution for designing an energy efficient fault tolerant optimum path WSN.

Our approach will work in two phases and both the phases will work in parallel for generating most efficient optimum path: In first phase, Genetic algorithms are applied on the current population set for finding an optimal solution and in parallel in the second phase Support Vector Machine will be applied on the set of nodes and it generates a report of nodes which have minimum energy and may leads GA to be failed; and passes the report to the GA so that the optimum result can be generated and energy can be saved.

\section{First Phase: Depth Genetic Algorithm (GA)}

- 'Define a sensor network with $\mathrm{N}$ number of nodes called $\left(\mathrm{SN}_{1}, \mathrm{SN}_{2}, \ldots, \mathrm{S}_{\mathrm{n}}\right)$ and 'm' number of targets $\left(\mathrm{t}_{1}, \mathrm{t}_{2}, \ldots, \mathrm{t}_{\mathrm{m}}\right)$.

- Apply Genetic algorithm to the current population of nodes for finding optimum path from parent node to the destination node.

- Apply fitness function to perform cost analysis.

- Implement crossover function K.

- $\quad$ Apply $\mathrm{K}=$ Mutation (K)

- If GA receives warning from SVM in second phase for any node in the current path which may lead in failure then GA leaves that node and randomly selects any other node for generating complete optimum path.

- $\quad$ Go to phase two.

Second Phase: Support Vector Machine (SVM)

- Define initial energy of each node with range and failure probability.

- $\quad$ Select the result of first phase as input vector $x_{i} \in R^{d}$, category $\mathrm{y}_{\mathrm{i}} \in\{-1,1\}$, sample size $\mathrm{I}, \mathrm{J}=1,2, \ldots, \mathrm{n}$.

- Apply Kernel function $\mathrm{K}\left(\mathrm{x}_{\mathrm{i}}, \mathrm{x}_{\mathrm{j}}\right)$, high dimensional feature space $\mathrm{Z}$, nonlinear mapping $\varphi: \mathrm{R}^{\mathrm{d}} \longrightarrow \mathrm{Z}$.

- In experiments, we select radial basis function (RBF) as the kernel function, and 250 rows of data as the training set. The optimal kernel parameters $\delta$ and penalty parameters $\mathrm{C}$ of seven one-class SVM classifier can be obtained in $\left(2^{-10}, 2^{15}\right)$ through grid search and 5-fold cross validation method. With the optimal parameters the classifier can be trained.

- If the result of first phase shows any node having minimum energy and which may leads in failure then SVM passes the information to the GA in first phase for changing the path.

- $\quad$ End

\section{CONCLUSION}

After reviewing some previously done important researches we have proposed a theoretical working system which is based on hybrid approach of using DFS based routing schemes with genetic algorithms for shortest connectivity path along with to find optimal result and then apply kernel function of support vector machine for detecting selective forwarding attack. Thus the paper concludes a system that can solve the issues of WSN which are coverage problem, optimum path, failure detection and energy efficient network.

\section{REFERENCES}

[1] "A Wireless Sensor Network- A Theoretical Review", Manoj Ahlawat, International Journal of Wired and Wireless Communications Vol.1, Issue 2, April, 2013.

[2] "Energy Efficient Wireless Sensor Network", Mrs. Rakhi Khedikar, Dr. Avichal Kapur, Dr. M.D. Chawhan, 2014 International Conference on Electronic Systems, Signal Processing and Computing Technologies,978-1-4799. 2102-7/14 (C) 2014 IEEE, DOI 10.1109/ICESC.2014.14

[3] "Coverage in Wireless Sensor Networks: A Survey", Raymond Mulligan, Habib M. Ammari, Network Protocols and Algorithms, 2010, Vol. 2, No. 2. 
[4] "N-target Coverage in Wireless Sensor Network using DFS and Genetic Algorithms", Anudeep, Subhash Chander, International Journal of Scientific Engineering and Research (IJSER), Volume 1 Issue 2, October 2013.

[5] "Energy-Efficient Target Coverage in Wireless Sensor Networks Based on Modified Ant Colony Algorithm",Salma Begum, Nazma Tara, Sharmin Sultana, International Journal of Ad hoc, Sensor \& Ubiquitous Computing (IJASUC) Vol.1, No.4, December 2010.

[6] "Maximizing Coverage in a Connected and K-Covered Wireless Sensor Network Using Genetic Algorithms", Tahir Emre Kalayc1, Kasım Sinan Y1ldırım, Aybars Ugur,International Journal Of Applied Mathematics And Informatics, Issue 3, Volume 1, 2007.

[7] "Review on Coverage and Connectivity in Underwater Wireless Sensor Networks", Manisha Yadav, Dr.(Prof.) Yusuf Mulge, International Journal of Computer Science \& Engineering Technology (IJCSET), Vol. 4 No. 05 May 2013.

[8] "Connectivity Issue in Wireless Sensor Networks by Using Depth-First Search and Genetic Algorithm",GholamAli Yaghoubi, 2010 International Conference on Computational Intelligence and Communication Systems 978-0-7695-4254-6/10 @ 2010 IEEE (pp 377- 381).

[9] "Malicious AODV: Implemen- tation and Analysis of Routing Attacks in MANETs", H. Ehsan and F. A. Khan,
IEEE Trust, Security and Privacy in Computing and Communications Conference TRUSTCOM, Liverpool, 25-27 June 2012, pp. 1181-1187.

[10] "Research on Intrusion Detection Algorithm Based on Multi-Class SVM in Wireless Sensor Networks", Hangxia Zhou, Qian Liu, Chen Cui, Communications and Network, 2013, 5, 524-528.

[11] "Support Vector Machines Based on K Nearest Neighbor Algorithm for Outlier Detection in WSNs", S. $\mathrm{Xu}, \mathrm{C}$. $\mathrm{Hu}, \mathrm{L}$. Wang and $\mathrm{G}$. Zhang, Proceedings of the 8th Wireless Communications, Networking and Mobile Com- puting International Conference WICOM, Shanghai, 21-23 September 2012, pp. 1-4.

[12] "Quarter-Sphere SVM: Attribute and Spatio-Temporal Correlations Based Outlier \& Event Detection in Wireless Sensor Networks", N. Shahid, I. H. Naqvi and S. B. Qaisar, IEEE Wireless Communications and Networking Conference WCNC, Shanghai, 1-4 April 2012, pp. 2048-2053.

[13] "Security Enhancement in Wireless Sensor Networks Using Machine Learning," A. B. Raj, M. V. Ramesh, R. V. Kulkarni and T. Hema- latha, High Performance Computing and Communication \& 2012 IEEE 9th International Conference on Embedded Software and Systems HPCC-ICESS, Liverpool, 25-27 June 2012, pp. 1264- 1269. 